\title{
Balance training using virtual reality improves
} balance and physical performance in older adults at high risk of falls

This article was published in the following Dove Press journal:

Clinical Interventions in Aging

\author{
Steven Phu (D) ${ }^{1,2}$ \\ Sara Vogrin (iD ${ }^{1,2}$ \\ Ahmed Al Saedi (iD) ${ }^{1,2}$ \\ Gustavo Duque (iD) ${ }^{1,2}$ \\ 'Department of Medicine, Melbourne \\ Medical School - Western Health, The \\ University of Melbourne, St. Albans, VIC, \\ Australia; ${ }^{2}$ Australian Institute for \\ Musculoskeletal Science (AIMSS), The \\ University of Melbourne and Western \\ Health, St. Albans, VIC, Australia
}

Purpose: Exercise programs designed for falls prevention have been proven effective in reducing falls by approximately $21 \%$. Virtual reality may provide a viable alternative intervention for falls prevention. This study compared the effects of virtual reality training using the Balance Rehabilitation Unit (BRU) versus exercise using a modified Otago Exercise Programme (EX) on improving balance and physical performance in the shortterm restorative care setting of the Gait and Balance Gym (Gabagym).

Patients and methods: This was a pre- and post-intervention study of 195 participants (median age 78 years, IQR 73-84; 67\% female) who presented with a risk and/or history of falls. Participants were assigned to either EX $(n=82)$ or BRU $(n=63)$. Supervised sessions occurred twice a week for 6 weeks. Participants receiving interventions were compared to a separate group $(\mathrm{n}=50)$ with similar characteristics who did not receive any intervention. Balance and physical performance were assessed at initial and final attendance and included the 5 Times Sit to Stand (5STS) test, Timed Up and Go (TUG), gait speed and posturography assessment using the BRU. Fear of falling was assessed using the Falls Efficacy Scale. Handgrip strength and adherence were also monitored.

Results: Post-intervention, EX and BRU groups achieved similar improvements and reported similar adherence rates $(71 \%$ vs $72 \%$, respectively). Both intervention groups improved in balance and physical performance measures. Both interventions showed significantly better improvement than the non-intervention group in TUG $(p<0.001)$, gait speed $(p=0.021)$, limits of stability in posturography assessment $(p=0.008)$, FES-I score $(p=0.013)$ and handgrip strength ( $p=0.021$ ). Only the BRU group improved control of static posture in the eyes closed $(p=0.002)$ and foam eyes closed $(p=0.006)$ tasks.

Conclusion: This study highlights the potential use of virtual reality as a practical alternative to improve outcomes of balance training for reduction of falls risk in older adults.

Keywords: posture, falls, fractures, virtual reality, exercise
Correspondence: Gustavo Duque Australian Institute for Musculoskeletal Science (AIMSS), Melbourne Medical School - Western Health, The University of Melbourne, 176 Furlong Road, St. Albans, VIC 302I, Australia

Tel +6I 383958121

Fax +61383958258

Email gustavo.duque@unimelb.edu.au

\section{Introduction}

Falls and the often-resulting fear of falling are major causes of disability in older adults by inducing reduced mobility and low quality of life. ${ }^{1}$ This is of concern given that falls occur in approximately $30 \%$ of older adults ${ }^{2}$ and are the leading cause of hospitalized injury in this population. ${ }^{3}$ Superficial injuries including bruising and cuts are common outcomes of falls, with approximately $4 \%$ resulting in a fracture (primarily attributed to osteoporosis). ${ }^{3}$ In the first three months after the hip fractures, 5-8 times higher mortality rate has been reported compared to 
those without hip fractures. ${ }^{4}$ Falls and fall-related injury evidently have an impact on individuals, family and their caregivers not withstanding costs to society and the health care system in general. ${ }^{5}$

Evidence of exercise for falls prevention is well established, ${ }^{6}$ with the Otago Exercise Programme (OEP) being an example of a well validated falls prevention program, ${ }^{7}$ which was initially designed as a home-based protocol but is also being effectively used in community programs. ${ }^{8}$ However, adherence to exercise programs in older adults is often low due to barriers including lack of motivation, ${ }^{9}$ enjoyment and fatigue. ${ }^{10}$

Interestingly, a recent systematic review has found higher adherence to technology-based programs ${ }^{11}$ including use of the WiiFit, Balance Rehabilitation Unit (BRU), and other computer-based systems. The BRU (Medicaa, Uruguay) is a virtual reality system used in the assessment and treatment of balance disorders by combining input from a force platform and virtual reality glasses containing a head tracker. ${ }^{12-17}$ It has been validated and found to be highly reliable when assessing balance in both younger and older participants with vestibular disorders. ${ }^{12}$ BRU has also been shown to be effective in reducing falls risk in high risk older persons, with improved control of static posture, reductions in measures for fear of falling and a decrease in fall rates demonstrated. ${ }^{13}$ This study aimed to provide further evidence for the effects of individualized virtual reality training using the BRU on balance and physical performance, and compare these results with a group exercise program using a community-based OEP in the clinical setting of the Gait and Balance Gym (Gabagym). We hypothesized that BRU intervention could not only improve static, but also transfer to dynamic balance.

\section{Materials and methods}

\section{Study population}

This was a pre- and post-intervention study of 195 community-dwelling older adults. Study participants were recruited from the local hospital, geriatricians and general practices across 2 sites in western Sydney (NSW, Australia) and western Melbourne (Sunshine, VIC, Australia). All participants were recruited using the same criteria as follows: aged over 65 years and self-reported balance deficits or a history of falls in the past year prior to assessment. A fall was defined as an "an unexpected event in which the participants come to rest on the ground, floor, or lower level". ${ }^{18}$ Further inclusion criteria consistent between the 2 sites included: being ambulant and able to mobilize independently (including the use of walking aids), absence of cognitive impairment (MMSE >24/30) and capacity to understand and follow simple instructions. Those who attended with a history of chronic disease (such as cardiovascular disease, diabetes, arthritis and osteoporosis) required clearance to exercise. Medical clearance was attained from the general practitioner who was provided with the American College of Sports Medicine for absolute and relative contraindications for exercise. ${ }^{19}$ Ethics approval was received from the Nepean Blue Mountains Local Health District Human Research Ethics Committee, and all participants provided written and informed consent prior to assessment and commencing their program. The study was conducted in accordance with the Declaration of Helsinki (1983) standards. Data across the 2 sites were collected between January 2013 and November 2015 (western Sydney) and January 2016 to February 2019 (western Melbourne) with the same individual conducting assessments and collecting data using identical equipment.

\section{Assessments}

Baseline assessment was conducted at both sites and involved an interview consisting of medical history, previous falls, symptoms and goals prior to taking part in the physical performance and posturography assessments. The Falls Efficacy Scale-International (FES-I) ${ }^{20}$ was administered to determine the participants subjective fear of falling with respect to activities of daily living. Height was measured with a stadiometer, and weight taken with a Tanita digital scale (Tanita Australia, Kewdale, WA, Australia). Body mass index (BMI) was calculated by dividing weight by the square of height in meters. All pre- and post-intervention assessments were conducted by the identical accredited exercise physiologist who had over 5 years of experience in developing interventions for falls prevention and was not blinded to the groups.

\section{Physical performance}

Assessments conducted in the study were determined based on factors including equipment required, time to perform, simplicity and sensitivity to changes in balance and physical performance. The assessments conducted included 5 times sit to stand (5STS), timed up and go (TUG), 4 square step test (FSST), gait speed, and handgrip strength. 
In the 5STS, participants were asked to stand and sit from a chair without any assistance or use of arms, 5 times as quickly as possible with the time to complete assessed. The chair was $40 \mathrm{~cm}$ in height, contained arm rests and was standardized and kept consistent for both initial and follow-up assessments. Participants were firstly instructed not to use chair arms to stand one time, with inability to do so marked as a failure to complete the test. Following successful performance of one STS, they were instructed to perform the test in full. A time of greater than or equal to $15 \mathrm{~s}$ was used to indicate falls risk. ${ }^{21}$

The TUG was completed from a chair $40 \mathrm{~cm}$ high (as previously described), with participants asked to stand and walk $3 \mathrm{~m}$ to a marked area at a normal pace, before turning and returning to the starting position with the time to complete measured. Participants were provided with 2 trials with the best time recorded. This study used a cutpoint of $13.5 \mathrm{~s}$ to determine falls risk. ${ }^{22}$

The 4-square step test assessed participants' change of direction ability, with participants asked to step in various directions throughout a marked course over a $2-\mathrm{cm}$ high block. The test was conducted following the protocol described by Dite and Temple, ${ }^{23}$ with a time to complete over $15 \mathrm{~s}$ indicating increased risk for falls. As with the TUG, 2 trials were provided for this test and the best time recorded.

Gait speed was assessed by having the participant walk a distance of $6 \mathrm{~m}$. A distance of $1 \mathrm{~m}$ was designated on each side for acceleration and deceleration. Gait speed was calculated using the time taken to walk the middle distance $(4 \mathrm{~m})$ at a normal pace. Participants were provided with 3 trials with the best gait speed used.

Handgrip strength was assessed using a Jamar Handgrip Dynamometer with the best of 3 trials recorded.

\section{Posturography assessment}

The posturography assessment was conducted using the BRU and involved participants standing on the BRU platform and undertaking several static tasks for $1 \mathrm{~min}$. Tasks included standing with: eyes open (firm surface) (EOEA); eyes closed (firm surface) (ECEA); standing on foam with eyes closed (FECEA); performing a saccadic task (SEA) and exposure to 2 visual-vestibular interaction tasks (VVIEA). The posturography assessment was completed within 30 mins. On completion, a posturography report was generated providing information regarding the limits of stability $\left(\mathrm{cm}^{2}\right)$, ellipse area/center of pressure area $\left(\mathrm{cm}^{2}\right)$ and sway velocity $(\mathrm{cm} / \mathrm{s})$ for each completed task. Limits of stability refers to the area in which an individual is able to shift his/her center of mass whilst maintaining balance and without changing the base of support (foot position), and is positively correlated with static balance, with older adults often presenting with reduced areas. ${ }^{24}$ Center of pressure and sway velocity measures were task-dependent, providing an insight into the individual's control of posture according to the balance system (visual, vestibular, proprioceptive) assessed. Participants displaying reduced limits of stability and larger center of pressure and sway velocity were identified as having greater balance impairment.

\section{Training modes}

Participants were placed into either exercise (EX) or virtual reality training (BRU) groups according to their initial assessment performance. Factors considered included patient safety, falls risk in a group exercise setting, medical history, performance in physical and balance assessments and clinical judgment. Participants with balance impairment (limits of stability below $170 \mathrm{~cm}^{2}$ ) and balance-related symptoms (ie, dizziness) were assigned to the BRU group. Both interventions were undertaken twice a week for 6 weeks, for a total of 12 sessions. Participants were assessed before and after completing the training program. Sessions were conducted and supervised by an accredited exercise physiologist who had received training in the modified OEP and BRU system.

\section{Exercise program}

Those assigned to the exercise program undertook a 60mins session which was based on the OEP, with exercises individually progressed. The OEP is a home-based exercise program which was designed to prevent falls in community dwelling older adults through strength and balance training. It has been previously validated in numerous studies, with significantly reduced falls and mortality demonstrated. ${ }^{25}$ In our Gabagym program, the OEP was modified where exercises were completed in a group setting following a circuit style, including a warm up and cool down pre/post exercise, and various stretching exercises. Additionally, the program duration was matched to that of the BRU program to ensure consistency in intervention programs. The group setting for exercise was chosen to allow for greater numbers of participants to be treated in a social environment to enhance adherence and has been proven as an effective mode of intervention. ${ }^{26}$ 


\section{Virtual reality balance training}

Virtual reality balance training was completed using the BRU system's preloaded programs for postural training and rehabilitation. BRU training followed a previously established protocol which included a rehabilitation component in addition to postural training games with each session lasting 30 mins. ${ }^{13}$ Briefly, the BRU software allowed for the creation of rehabilitation exercises according to individual needs which were based on exercises performed in the posturography assessment. Rehabilitation exercises included tasks performed standing with virtual reality headset on whereby the visual and vestibular systems were stressed and could be customized according to the participant's needs and ability to tolerate the training stimulus. Variables which could be modified included time of exposure, rate/frequency and surface. Postural training exercises were used to develop limits of stability, with 3 exercises performed requiring participants to move their center of mass to collect rings/blocks through a course within a given amount of time. Each of the 3 postural training exercises contained 15 levels of varying difficulty. Participants undertook a combination of rehabilitation and postural training exercises, with approximately 15 mins spent in each. Sessions were tailored to each individual, with progression achieved by increasing the level of difficulty or time of exposure for each exercise.

\section{Non-intervention group}

Participants in the group who did not receive BRU or EX were recruited from western Melbourne between January 2016 and February 2019 and fulfilled the inclusion criteria as previously described. As with the intervention groups, these participants were recommended to attend the EX or BRU programs; however, they did not. Participants undertook the same assessments as the intervention groups using identical equipment and were provided with education regarding their falls risk. At follow-up, participants did not report performing any form of exercise.

\section{Statistical analysis}

The majority of data obtained from participants were not normally distributed; therefore, median and interquartile ranges are presented for all measures to ensure consistency. Baseline characteristics are presented as frequency (percentage) for categorical variables and median (interquartile range) for continuous variables. Between-group differences were assessed using Fisher's exact tests and Kruskal Wallis test due to non-normal distribution of continuous variables.
General estimating equations were used to examine the changes within groups with an interaction term between time and group to evaluate whether the effect of intervention differed. All variables with the exception of gait speed required log transformation. Statistical analyses were conducted using Stata 13.1 (StataCorp. 2013. Stata Statistical Software: Release 13. College Station, TX: StataCorp LP.).

\section{Results}

Data from 195 participants were collected in our analysis (median age 78 years, IQR: $73-84,67 \%$ female). After the initial assessment, 63 (32\%) participants were allocated to the BRU group and $82(42 \%)$ participants undertook exercise-based balance training. The non-intervention "control" group contained 50 participants (26\%).

The baseline characteristics for each group are shown in Table 1. No significant between-group differences were found at baseline for demographics, physical and posturography measures except for the mobility assessments, where those in the exercise group performed better compared to the control and BRU groups for the TUG ( $p=0.029$ and $p=0.002$, respectively) and gait speed ( $p=0.004$ and $p=0.001$, respectively).

After completing the program, the intervention groups showed a significant improvement in performance for all physical performance and subjective measures (5STS, TUG, FSST, gait speed, FES-I and handgrip strength), whereas the control group did not report improvements in TUG, FES-I score and handgrip strength (Table 2). Significant differences between the 3 groups for physical performance and fear of falling (TUG, gait speed, FES-I score and handgrip strength) were evident (Table 2), with a comparison of groups shown in Figure 1. In balance measures, both intervention groups achieved significant improvements of at least $20 \%$ for limits of stability (BRU $p<0.001, \mathrm{EX} p=0.01$ ) and FECEA tasks (BRU $p=0.006$, EX $p=0.04$ ), with small but non-significant changes observed for the control group (Table 3). Only the BRU group achieved significant gains in ECEA task ( $p=0.001)$. No significant differences were observed for the EOEA and SEA tasks (Table 3). A comparison of changes between groups can be found in Figure 2 .

Adherence was similar between the 2 intervention groups, with the EX group recording rates of $72 \%$ compared to the BRU group of $71 \%$.

\section{Discussion}

With the emerging and increasing use of technology in the exercise and rehabilitation setting, the aim of this study 
Table I Baseline characteristics of included participants reported as median (IQR)

\begin{tabular}{|c|c|c|c|c|}
\hline Baseline characteristics & BRU, $N=63$ & Exercise, $\mathbf{N}=82$ & Control, $\mathbf{N}=\mathbf{5 0}$ & Between-group p-value \\
\hline Age, y & $79(74,84)$ & $76(71,82)$ & $79(72,82)$ & 0.37 \\
\hline Sex & & & & 0.55 \\
\hline Male, n (\%) & $19(30.2)$ & $31(37.8)$ & $15(30 \%)$ & \\
\hline Female, n (\%) & $44(69.8)$ & $51(62.2)$ & $35(70 \%)$ & \\
\hline Body mass index, $\mathrm{kg} / \mathrm{m}^{2}$ & $27.0(25.0,31.0)$ & $27.0(23.5,31.0)$ & $28.5(24.1,32.6)$ & 0.60 \\
\hline Handgrip strength $(\mathrm{kg})$ & $19(16,25)$ & $20.5(17,27)$ & $22(16,28)$ & 0.42 \\
\hline 5 Sit to Stand, s & $21.1(16.9,28.8)$ & $18.5(15.4,26.4)$ & $17.8(14.8,22.9)$ & 0.11 \\
\hline Timed Up and Go, s & $15.0(11.8,19.4)$ & $11.9(10.0,15.8)$ & $16.0(10.2,21.8)$ & 0.005 \\
\hline Gait Speed, m/s & $0.78(0.58,0.91)$ & $1.03(0.80,1.13)$ & $0.80(0.52,0.98)$ & 0.002 \\
\hline Limits of Stability, $\mathrm{cm}^{2}$ & III (80, 144) & $132(9|| 56)$, & $126(53,161)$ & 0.55 \\
\hline Falls Efficacy Scale - International (16-64) & $34(27,39)$ & $33(25,4 I)$ & $32(24,43)$ & 0.93 \\
\hline
\end{tabular}

Abbreviation: BRU, balance rehabilitation unit.

was to evaluate the effects of a virtual reality system on balance and physical performance in community-dwelling older adults compared to a modified $\mathrm{OEP}^{7,25}$ in the shortterm restorative care setting of the Gabagym.

BRU has been previously described as a form of training which stresses the balance and visual-vestibular systems in a manner which is not physically taxing. ${ }^{13}$ In addition to this, the one-on-one nature of BRU allowed participants to receive individually tailored treatment for their balance deficits, which may further enhance improvements and adherence. A recent systematic review suggested inconclusive effects of virtual reality on physical function; however, the majority of studies included in the review used the Nintendo Wii gaming console which is not specifically designed for balance training as the BRU system is. ${ }^{27}$ This study highlights the effectiveness of the virtual reality BRU system in improving static and dynamic balance, and physical performance in community-dwelling older adults. Improvements in posturography mirrored that of an earlier study which also found reduced fear of falling and fall rates over a 9-month period. ${ }^{13}$ However, the previous study did not provide a comparison to other validated programs for falls prevention such as our modified OEP. In addition to the improved posturography, this study also found significant gains in physical performance such as handgrip strength, 5STS, TUG, FSST and gait speed. This may suggest that BRU training, which is static in nature, may have translatable improvements to dynamic balance.
The use of exercise as an intervention for falls prevention has been well established in the research, including a Cochrane review finding that the most effective program for fall prevention was multi-component, including the use of strengthening and balance training programs. ${ }^{7}$ Program design is particularly important with recommendations stating that exercises with a moderate or high balance challenge should be undertaken a minimum of 2 hrs per week on a continuing basis. ${ }^{6,28}$ The exercise program used in this study adhered to well-established recommendations ${ }^{7}$ which may explain the significantly improved physical performance and posturography results. Of note is the fact that the BRU group was able to achieve similar physical improvements to that of the EX group, despite halved training time. This was surprising given the nature of BRU training and may be explained by potential ceiling effects in the EX group considering the baseline significant differences in TUG and gait speed. Despite the similar improvements, there is a possibility that the EX group may have achieved greater improvements which were not evident due to baseline imbalances caused by a non-random allocation.

Poor falls efficacy and low confidence in balance can inhibit the performance of activities of daily living, lead to cycle of reduced physical capacity, loss of independence and further falls. ${ }^{29}$ Our study utilized the FES-I as a measure of the impact fear of falls presents on completion of daily activities and falls efficacy. We found a significant reduction in the FES-I in both groups of at least $10 \%$ which was similar to the modest impacts of exercise 


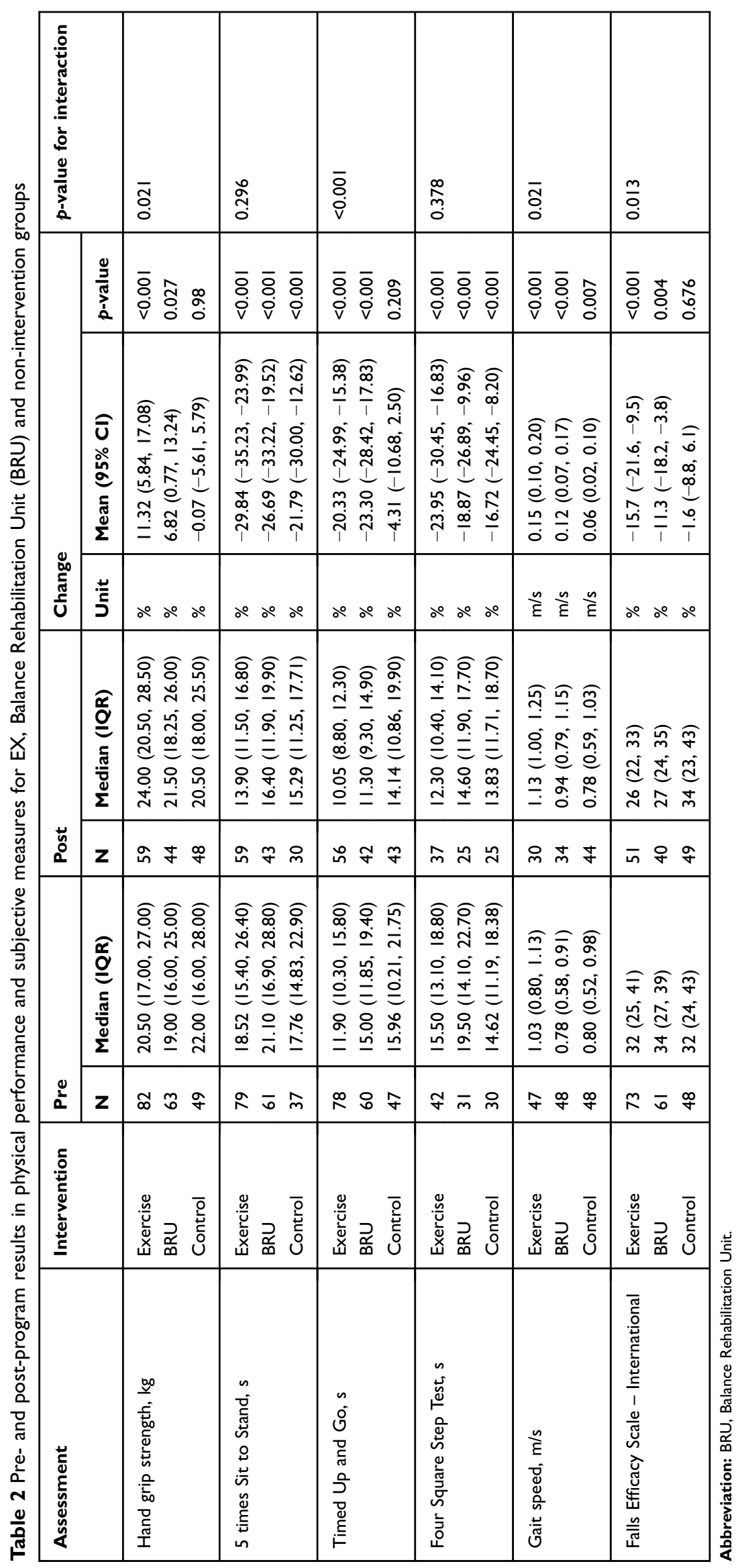




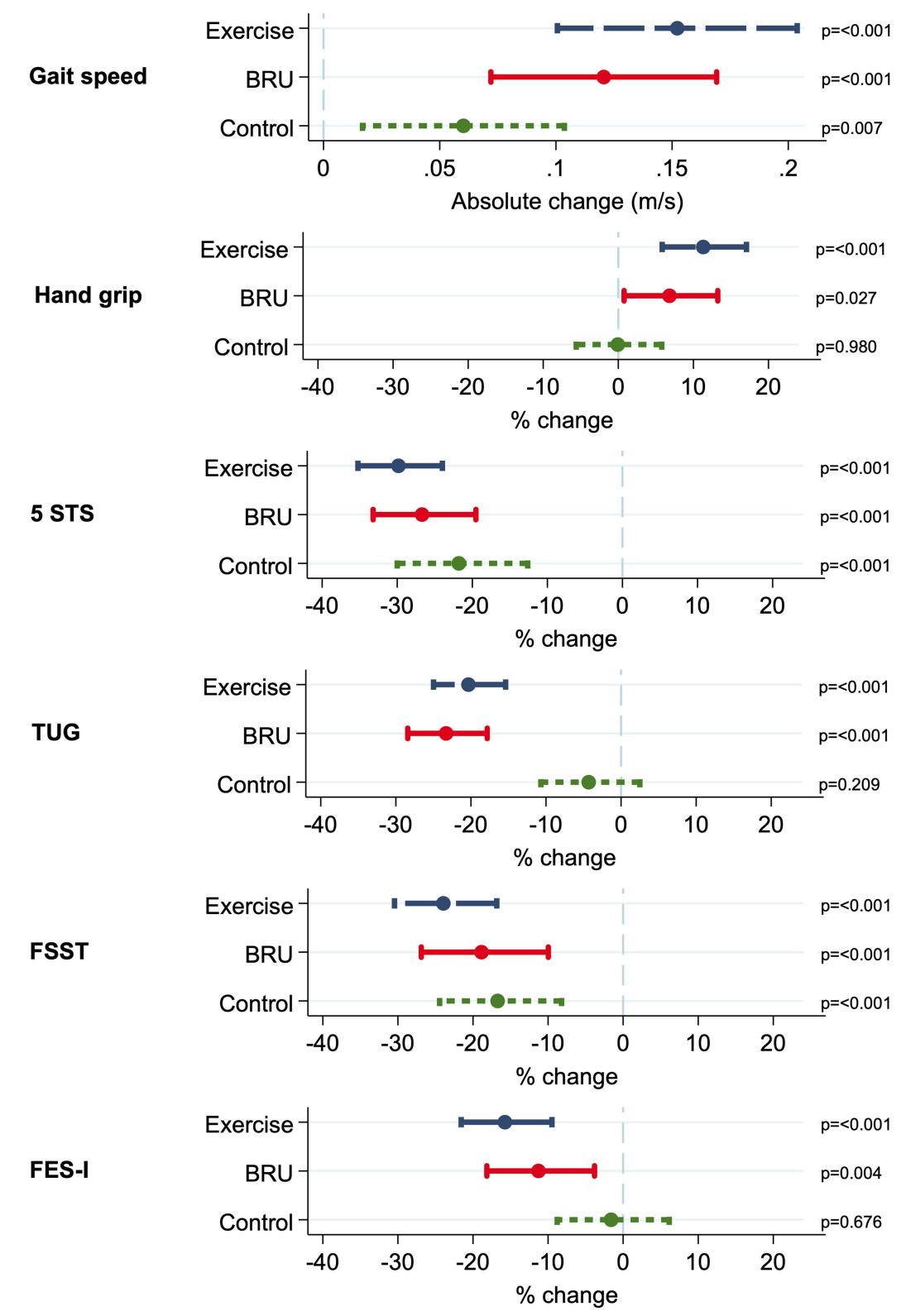

Figure I Percentage change for handgrip strength, five time sit to stand (5 STS), timed up and go (TUG), four square step test (FSST) and falls efficacy scale (FES-I) assessments. Absolute change included for gait speed. $p$-values for comparisons between pre and post intervention are listed on the right hand side for each intervention. Significant difference was found between the exercise and BRU groups compared to control (non-intervention) for handgrip strength, TUG, gait speed and fear of falling (FES-I) assessments.

reported in a recent systematic review. ${ }^{29}$ Despite the larger absolute improvements for the majority of physical performance measures in the exercise group, no betweengroup differences were evident with BRU, suggesting that BRU may be as effective as exercise in improving physical performance and falls risk in older adults.

\section{Limitations}

There were several limitations to this study. Firstly, given the observational nature of the study, we did not control for activities performed in addition to the supervised sessions including exercise. Hence, it was possible that participants were able to increase their activity levels as their physical performance and fear of falling improved, resulting in further increases post intervention.

Secondly, participants were not randomized, but the group allocation was based on initial balance and physical performance assessment. Given this study did not randomize the allocation of participants to intervention, there is the possibility for the introduction of bias to the results due to the occurrence of regression to the mean. Regression to the mean is a concern for all studies which include 


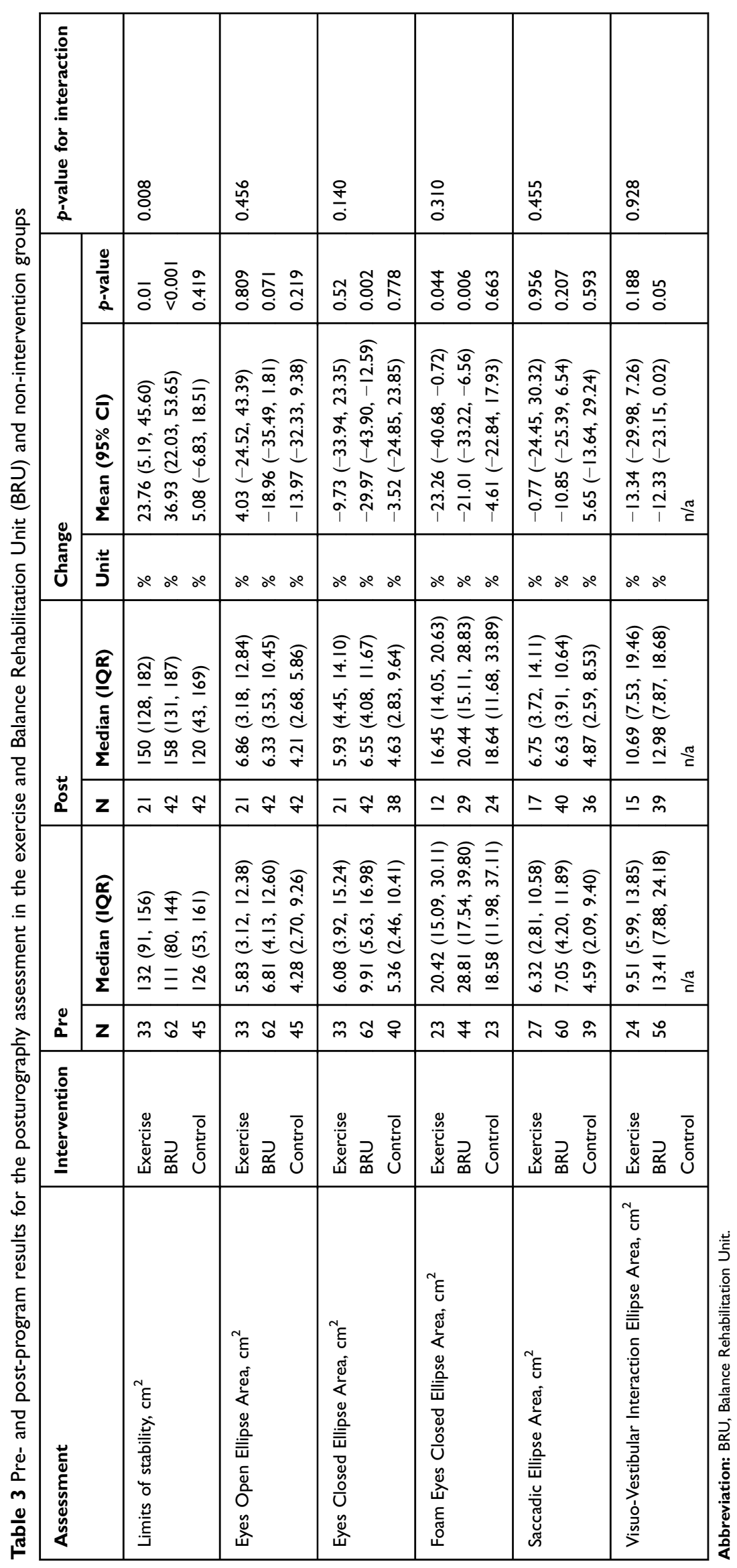


Los

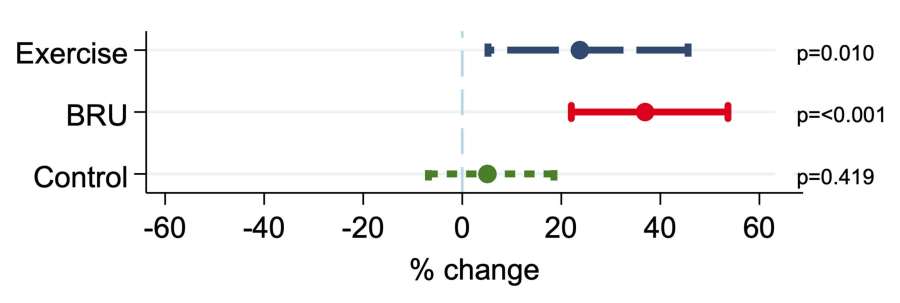

EOEA

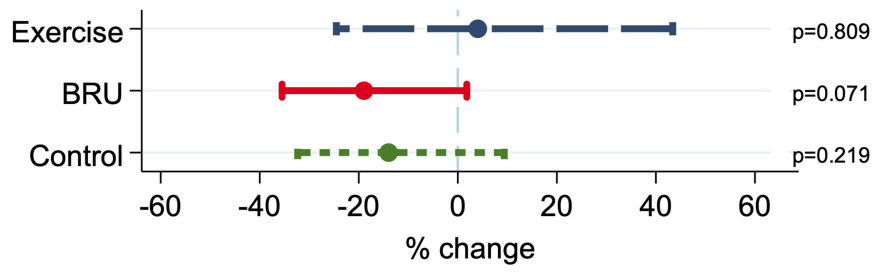

ECEA

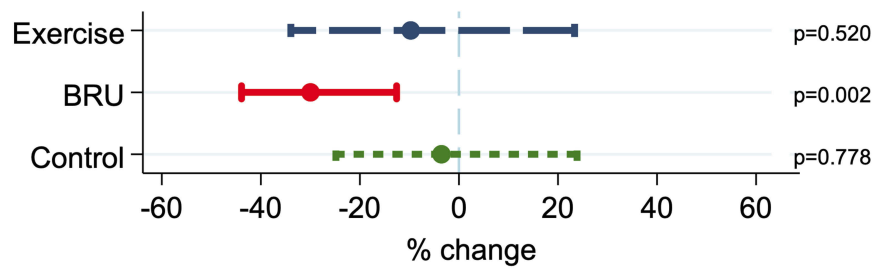

FECEA

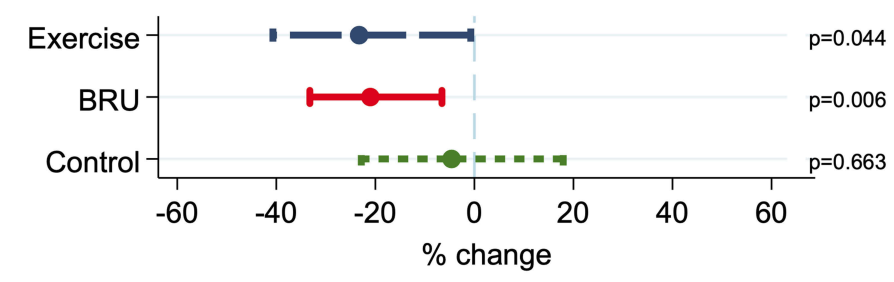

SEA
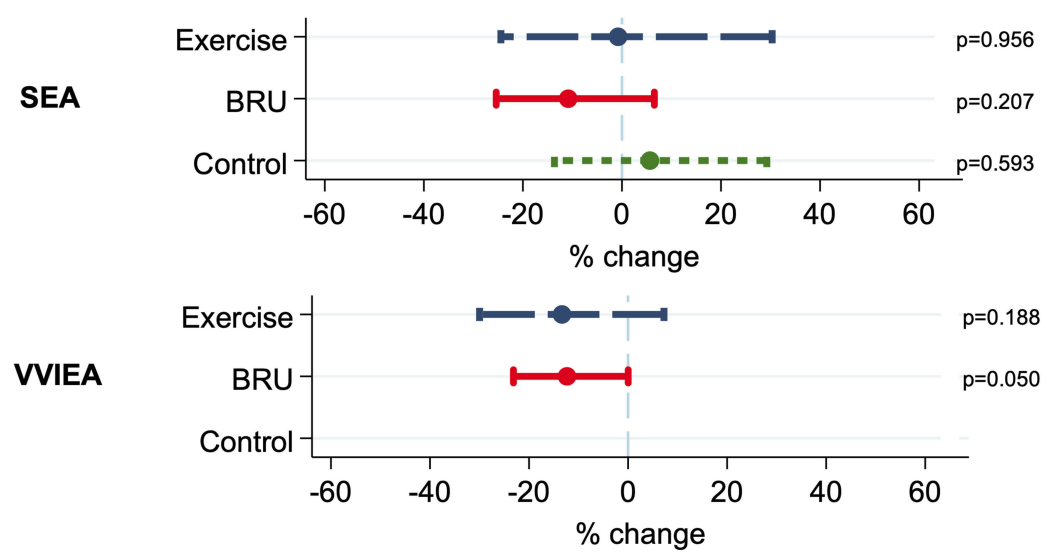

Figure 2 Percentage change for the posturography assessment results for limits of stability (LOS) and ellipse areas for the following tasks: eyes open (EOEA), eyes closed task (ECEA), foam eyes closed (FECEA), saccadic (SEA), visuo-vestibular interaction (VVIEA). p-values for comparisons between pre and post intervention are listed on the right hand side for each intervention. Although the BRU group reported larger and more significant changes, only improvements in limits of stability were significantly different between groups.

Abbreviation: BRU, Balance Rehabilitation Unit.

repeated measures whereby random error at assessment may result in the incorrect interpretation of results. As this study could not address regression to the mean by randomizing participants to groups, we have ensured that the assessment results would accurately reflect performance through the use of multiple trials for each assessment to allow for familiarization, followed by recording the best performance in each trial as opposed to the mean.
In some ways, this has reduced the significant difference between groups for all assessments (with the exception of TUG and gait speed). Despite this, groups were well balanced on demographic, physical performance and clinical characteristics. In addition, the control group which was included was recruited from a different population (western Melbourne). This may be a confounding factor; however, the inclusion criteria and baseline characteristics 
were largely consistent between groups with no significant differences between groups at baseline for the majority of measures.

Third, the cross-sectional short-term nature of this study did not allow us to quantify falls in the long term, which could be a potential outcome in future longitudinal studies. As a strength of this study, we have tested a new technology specifically designed to measure and treat balance in a population of participants at high risk of falls and fractures. In addition, few studies can offer the breadth of physical performance and clinical characteristics available in this study for use in understanding the effect of virtual reality on falls risk.

\section{Conclusion}

Falls prevention programs involving the use of exercise have been extensively studied in the literature and shown to be effective. The findings of our study have demonstrated the potential benefits of virtual reality balance training on balance and physical performance, factors important for falls prevention. When compared to OEP, the BRU was found to produce similar results to that of exercise. Given that there is often a void in short-term restorative care programs providing treatment between an individual having a fall and returning to daily life in the community, this study provides a basis for future research by highlighting the possibilities of virtual reality balance training to improve balance and physical performance, to a similar degree as traditional exercise. This may particularly benefit those who present with contraindications for or are reluctant and/or non-adherent to conventional exercise programs.

\section{Acknowledgments}

The authors would like to thank the Australian Institute for Musculoskeletal Science (AIMSS) and Melbourne Medical School for providing support to this study. This study was funded by the Australian Institute for Musculoskeletal Science (AIMSS).

\section{Author contributions}

All authors contributed to data analysis, drafting or revising the article, gave final approval of the version to be published, and agree to be accountable for all aspects of the work.

\section{Disclosure}

The authors report no conflicts of interest in this work.

\section{References}

1. Patil R, Uusi-Rasi K, Kannus P, Karinkanta S, Sievanen H. Concern about falling in older women with a history of falls: associations with health, functional ability, physical activity and quality of life. Gerontology. 2014;60(1):22-30. doi:10.1159/000354335

2. Morrison A, Fan T, Sen SS, Weisenfluh L. Epidemiology of falls and osteoporotic fractures: a systematic review. Clinicoecon Outcomes Res. 2013;5:9-18. doi:10.2147/CEOR.S38721

3. Tovell A, Harrison J, Pointer S. Hospitalised Injury in Older Australians, 2011-12. In: AIHW, editor. Canberra: AIHW; 2014.

4. Haentjens P, Magaziner J, Colon-Emeric CS, et al. Meta-analysis: excess mortality after hip fracture among older women and men. Ann Intern Med. 2010;152(6):380-390. doi:10.7326/0003-4819-152-6-201003160-00008

5. Heinrich S, Rapp K, Rissmann U, Becker C, Konig HH. Cost of falls in old age: a systematic review. Osteoporos Int. 2010;21(6):891-902. doi:10.1007/s00198-009-1100-1

6. Sherrington C, Michaleff ZA, Fairhall N, et al. Exercise to prevent falls in older adults: an updated systematic review and meta-analysis. Br J Sports Med. 2017;51(24):1750-1758. doi:10.1136/bjsports-2016-096547

7. Gillespie LD, Robertson MC, Gillespie WJ, et al. Interventions for preventing falls in older people living in the community. Cochrane Database Syst Rev. 2012;(9):CD007146.

8. Waters DL, Hale LA, Robertson L, Hale BA, Herbison P. Evaluation of a peer-led falls prevention program for older adults. Arch Phys Med Rehabil. 2011;92(10):1581-1586. doi:10.1016/j.apmr.2011.05.014

9. Forkan R, Pumper B, Smyth N, Wirkkala H, Ciol MA, Shumway-Cook A. Exercise adherence following physical therapy intervention in older adults with impaired balance. Phys Ther. 2006;86(3):401-410.

10. Rhodes RE, Martin AD, Taunton JE, Rhodes EC, Donnelly M, Elliot J. Factors associated with exercise adherence among older adults. An individual perspective. Sports Med. 1999;28(6):397-411. doi:10.2165/ 00007256-199928060-00003

11. Valenzuela T, Okubo Y, Woodbury A, Lord SR, Delbaere K. Adherence to technology-based exercise programs in older adults: a systematic review. J Geriatr Phys Ther. 2018;41(1):49-61. doi:10.1519/JPT.0000000000000095

12. Alahmari KA, Marchetti GF, Sparto PJ, Furman JM, Whitney SL. Estimating postural control with the balance rehabilitation unit: measurement consistency, accuracy, validity, and comparison with dynamic posturography. Arch Phys Med Rehabil. 2014;95(1):65-73. doi:10.1016/j.apmr.2013.09.011

13. Duque G, Boersma D, Loza-Diaz G, et al. Effects of balance training using a virtual-reality system in older fallers. Clin Interv Aging. 2013;8:257-263. doi:10.2147/CIA.S41453

14. Suarez H, Geisinger D, Ferreira ED, et al. Balance in Parkinson's disease patients changing the visual input. Braz J Otorhinolaryngol. 2011;77(5):651-655.

15. Dona F, Aquino CC, Gazzola JM, et al. Changes in postural control in patients with Parkinson's disease: a posturographic study. Physiotherapy. 2016;102(3):272-279. doi:10.1016/j.physio.2015.08.009

16. Kasse CA, Santana GG, Branco-Barreiro FC, et al. Postural control in older patients with benign paroxysmal positional vertigo. Otolaryngol Head Neck Surg. 2012;146(5):809-815. doi:10.1177/0194599811434388

17. Cusin FS, Gananca MM, Gananca FF, Gananca CF, Caovilla HH. Balance Rehabilitation Unit (BRU) posturography in Meniere's disease. Braz J Otorhinolaryngol. 2010;76(5):611-617.

18. Lamb SE, Jorstad-Stein EC, Hauer K, Becker C, Prevention of Falls Network E, Outcomes Consensus G. Development of a common outcome data set for fall injury prevention trials: the Prevention of Falls Network Europe consensus. J Am Geriatr Soc. 2005;53 (9):1618-1622. doi:10.1111/j.1532-5415.2005.53455.x

19. Arena R. Exercise Testing. In: Pescatello L, editor. American College of Sports Medicine Guidelines for Exercise Testing and Prescription. Philadelphia, PA: Wolters Kluwer/Lippincott Williams and Wilkins Health; 2014:53. 
20. Yardley L, Beyer N, Hauer K, Kempen G, Piot-Ziegler C, Todd C. Development and initial validation of the Falls Efficacy ScaleInternational (FES-I). Age Ageing. 2005;34(6):614-619. doi:10.1093/ageing/afi196

21. Buatois S, Perret-Guillaume C, Gueguen R, et al. A simple clinical scale to stratify risk of recurrent falls in community-dwelling adults aged 65 years and older. Phys Ther. 2010;90(4):550-560. doi: $10.2522 / \mathrm{ptj} .20090158$

22. Shumway-Cook A, Brauer S, Woollacott M. Predicting the probability for falls in community-dwelling older adults using the timed up \& go test. Phys Ther. 2000;80(9):896-903.

23. Dite W, Temple VA. A clinical test of stepping and change of direction to identify multiple falling older adults. Arch Phys Med Rehabil. 2002;83(11):1566-1571.

24. Horak FB. Postural orientation and equilibrium: what do we need to know about neural control of balance to prevent falls? Age Ageing. 2006;35(Suppl 2):ii7-ii11. doi:10.1093/ageing/afl077
25. Thomas S, Mackintosh S, Halbert J. Does the 'Otago exercise programme' reduce mortality and falls in older adults?: a systematic review and metaanalysis. Age Ageing. 2010;39(6):681-687. doi:10.1093/ageing/afq102

26. Kyrdalen IL, Moen K, Roysland AS, Helbostad JL. The Otago Exercise Program performed as group training versus home training in fall-prone older people: a randomized controlled trial. Physiother Res Int. 2014;19(2):108-116. doi:10.1002/pri.1571

27. Molina KI, Ricci NA, de Moraes SA, Perracini MR. Virtual reality using games for improving physical functioning in older adults: a systematic review. J Neuroeng Rehabil. 2014;11:156. doi:10.1186/ 1743-0003-11-156

28. Sherrington C, Fairhall NJ, Wallbank GK, et al. Exercise for preventing falls in older people living in the community. Cochrane Database Syst Rev. 2019;1:CD012424. doi:10.1002/14651858.CD005619.pub3

29. Rand D, Miller WC, Yiu J, Eng JJ. Interventions for addressing low balance confidence in older adults: a systematic review and meta-analysis. Age Ageing. 2011;40(3):297-306. doi:10.1093/ageing/afr037
Clinical Interventions in Aging

\section{Publish your work in this journal}

Clinical Interventions in Aging is an international, peer-reviewed journal focusing on evidence-based reports on the value or lack thereof of treatments intended to prevent or delay the onset of maladaptive correlates of aging in human beings. This journal is indexed on PubMed Central, MedLine, CAS, Scopus and the Elsevier
Dovepress

Bibliographic databases. The manuscript management system is completely online and includes a very quick and fair peer-review system, which is all easy to use. Visit http://www.dovepress.com/ testimonials.php to read real quotes from published authors. 